\title{
Efficient implementation of finite volume methods in Numerical Relativity
}

\author{
Daniela Alic, Carles Bona, Carles Bona-Casas and Joan Massó \\ Departament de Fisica, Universitat de les Illes Balears, Palma de Mallorca, Spain
}

\begin{abstract}
Centered finite volume methods are considered in the context of Numerical Relativity. A specific formulation is presented, in which third-order space accuracy is reached by using a piecewise-linear reconstruction. This formulation can be interpreted as an 'adaptive viscosity' modification of centered finite difference algorithms. These points are fully confirmed by $1 \mathrm{D}$ black-hole simulations. In the $3 \mathrm{D}$ case, evidence is found that the use of a conformal decomposition is a key ingredient for the robustness of black hole numerical codes.

PACS numbers: 04.25.Dm
\end{abstract}

\section{INTRODUCTION}

In recent times, many numerical relativity groups have performed long-term binary-black-hole (BBH) simulations. This was a long sought goal, with the main objective of computing gravitational wave patterns that can be used as templates for detection. The $\mathrm{BBH}$ case was specially relevant in this respect because it is assumed to be the most likely candidate for detection by the current ground-based interferometric facilities. This can explain why the focus in these simulations has been placed in the accurate modelling of the wave zone: the numerical boundaries are placed safely far away, which implies the use of large computational domains. Also, the ability to extract the gravitational wave signal from the wave zone evolution requires the simulation to last for quite a long time. These facts, together with the use of some form of mesh refinement in order to ensure the required accuracy, make BBH simulations very demanding for the computational point of view, requiring a big computing infrastructure.

Black hole simulations, however, deserve some interest by themselves, independently of the quest for gravitational waves. One can focus for instance on the strong field region, which can be modelled by using modest-size computational domains. In this case, one must refrain from excising the black hole interior, although many interesting results have been obtained by using excision [1], even in cases with some matter content [2]. The consequences of this choice are well known (see Ref. [3] for a very clear recent example):

- A singularity-avoidant gauge condition must be used in order to prevent a singularity to form inside the computational domain in a finite amount of coordinate time.

- This makes the lapse to collapse in the black hole interior zones, while keeping its initial profile in the outer region.

- As a consequence, steep lapse gradients appear near the apparent horizon, which challenge the stability of the numerical algorithm.

Most of the current BH simulations are performed with finite difference algorithms. Regarding space accuracy, the most common approach is to use a centered fourthorder accurate method, combined with some artificial dissipation term (Kreiss-Oliger dissipation) [4]. The leading error in the solution is precisely the artificial dissipation one, usually of fourth order. One can interpret this combination just as a particular third-order scheme with some built-in dissipation, which can be tuned by a single parameter. This may be a difficulty in some cases, where dealing with the black hole interior would require an amount of dissipation which can be instead too big for the exterior region (see for instance Ref. [3]). Our point is that centered Finite Volume methods can provide alternative third-order accurate algorithms in which the built-in dissipation is automatically adapted to the requirements of either the interior or exterior black hole regions.

Finite Volume (FV) methods have a reputation of being computationally expensive, a price that is not worth to pay for spacetime simulations, where the dynamical fields usually have smooth profiles. From this point of view, centered FV methods can provide some improvement, because the they do not require the full characteristic decomposition of the set of dynamical fields: only the values of the propagation speeds are needed [5].

This point can be illustrated by comparing the classical FV techniques implemented in a previous work [6] (hereafter referred as paper I) with the new FV methods presented here. In paper I, the general relativistic analogous of the Riemann problem must be solved at every single interface. This implies transforming back and forth between the primitive variables (the ones in which the equations are expressed) and the characteristic ones (the eigenvectors of the characteristic matrix along the given axis). In the present paper, a simple flux formula is applied directly on the primitive variables, so that switching to the characteristic ones is no longer required. The flux formula requires just the knowledge of the characteristic speeds, not the full decomposition.

Another important difference is that in paper I, the primitive quantities where reconstructed from their average values in a piecewise linear way, using a unique slope at every computational cell. Only (piecewise) second order accuracy can be achieved in this way, so that going to (piecewise) third order would require the use of 'piecewise parabolic methods' (PPM), with the corresponding com- 
putational overload. In this paper instead we split every flux into two components before the piecewise-linear reconstruction (flux-splitting approach [5]). This allows using a different slope for every flux component: this extra degree of freedom allows us to get (piecewise) third order accuracy for a specific choice of slopes, without using PPM.

It is true that third-order convergence is rarely seen in practice. In the context of Computational Fluid Dynamics (CFD), this is due to the arising of physical solutions (containing shocks or other discontinuities) which are just piecewise smooth. These discontinuities can propagate across the computational domain and the convergence rate is downgraded as a result in the regions swept away by the discontinuity front. A similar situation is encountered in black hole evolutions. The use of singularity avoidant slicing conditions produces a collapse in the lapse function. As it can be seen in Fig. 2, a steep gradient surface is formed (the collapse front) that propagates out as the grid points keep falling into the black hole. We will see that this results into a downgrade of accuracy in the regions close to the collapse front.

Stability problems can also arise from the lack of resolution of the collapse front, which is typically located around the apparent horizon. The reconstruction procedure can lead there to spurious oscillations, which introduce high-frequency noise in the simulation. In paper I, this problem was dealt with the use of standard slope limiters, which were crucial for the algorithm stability. In the present paper, although slope limiters are also discussed for completeness, their use is not even required in any of the presented simulations. The new algorithm gets rid by itself of the high-frequency noise, even for the steep (but smooth) profiles appearing around the blackhole horizon.

With all these simplifications, the proposed centered FV method can be interpreted just as an 'adaptive viscosity' generalization of the finite difference (FD) algorithms discussed before. Moreover, in the FV context, boundary conditions can be imposed in a simple way by the 'ghost point' technique. This allows one to avoid the complications related to the corners and edges treatment that usually appear in the FD context.

The paper is organized as follows: we present in Section II a brief summary of the simplest FV methods. In Section III, the flux-splitting variant is considered, and we show how third-order space accuracy can be obtained by using just linear reconstruction. The resulting method is then tested for the one-dimensional (1D) black-hole in Section IV. Long term (up to $1000 \mathrm{~m}$ ) simulations are performed with a single numerical grid of a limited resolution, showing the efficiency of the algorithm. A convergence test is also performed, which confirms the predicted third-order accuracy in the outside region. The threedimensional (3D) black-hole case is considered in Section V. A low resolution simulation is presented, showing the key role of controlling the trace of the extrinsic curvature in order to avoid numerical instabilities. This explains the advantage of using tr $K$ as a primitive variable, like in the Conformal ADM (CADM) formalism [7]. This explains also why a conformal decomposition was also required for obtaining robust 3D simulations in paper I, even when using FV methods [6].

For the sake of clarity, the more technical points: stability analysis, time evolution algorithms and the full explicit form of the equations, are described in Appendices $\mathrm{A}, \mathrm{B}$ and $\mathrm{C}$, respectively.

\section{CENTERED FINITE VOLUME METHODS: FLUX FORMULAE}

Let us consider the well known $3+1$ decomposition of Einstein's field equations. The extrinsic curvature $K_{i j}$ is considered as an independent dynamical field, so that the evolution system is of first order in time but second order in space. Let us transform it into a fully first order system by considering also the first space derivatives of the metric as independent quantities. This requires additional evolution equations for these space derivatives, that can be obtained in the standard way by permuting space and time derivatives of the metric, that is

$$
\partial_{t}\left(\partial_{k} g_{a b}\right)=\partial_{k}\left(\partial_{t} g_{a b}\right)
$$

so that the resulting first order system will describe the same dynamics than the original second order one.

In this first order form, Einstein's field equations can always be expressed as a system of balance laws [8]. The evolution system can be written in the form

$$
\partial_{t} \mathbf{u}+\partial_{k} \mathbf{F}^{k}(\mathbf{u})=\mathbf{S}(\mathbf{u}),
$$

where both the Flux terms $\mathbf{F}$ and the Source terms $\mathbf{S}$ depend algebraically on the array of dynamical fields $\mathbf{u}$, which contains the metric and all its first derivatives. The terms 'Fluxes' and 'Sources' come from the hydrodynamical analogous of the system (2).

The balance law form is well suited for FV discretization methods. The idea is to evolve the average of the dynamical fields $\mathbf{u}$ on some elementary cells, instead of evolving just point values like in the FD approach. The space discretization can be obtained by averaging (2) over an elementary cell and applying the divergence theorem to get:

$$
\partial_{t} \overline{\mathbf{u}}+\oint \mathbf{F}^{k} d S_{k}=\overline{\mathbf{S}}
$$

where the overlines stand for space averages. The evaluation of partial space derivatives has been replaced in this way by that of surface integrals of the flux terms.

Let us consider for simplicity the one-dimensional case. We can start from a regular finite difference grid. The elementary cell can then be chosen as the interval $\left(x_{i-1 / 2}, x_{i+1 / 2}\right)$, centered on the generic grid point $x_{i}$. The dynamical fields $\mathbf{u}$ can be modelled as piecewise linear functions in every cell (linear reconstruction, see 


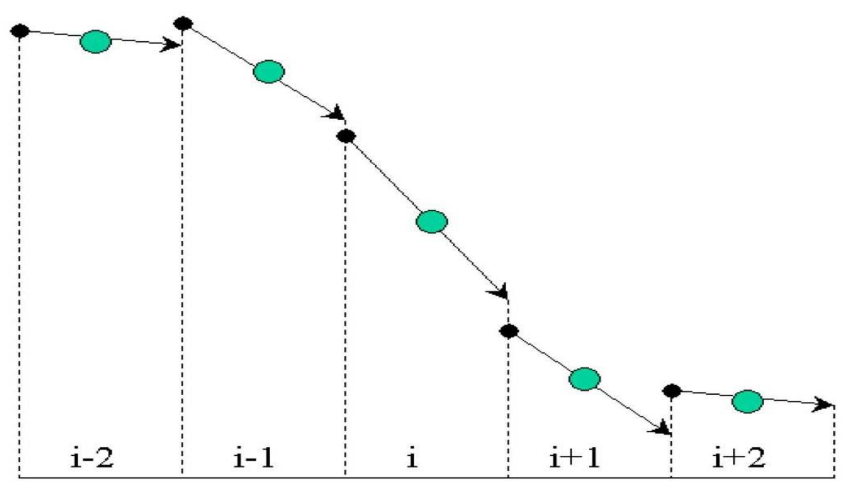

FIG. 1: Piecewise linear reconstruction of a given function. Numerical discontinuities appear at every cell interface (dotted lines) between the left and right values (arrows and dots, respectively). Note that the original function was monotonically decreasing: all the slopes are negative. However, both the left interface values (at $i+3 / 2)$ and the right interface ones (at $i-3 / 2$ ) show local extremes that break the monotonicity of the original function.

Fig. 1), so that the average values $\overline{\mathbf{u}}_{i}$ coincide with the point values $\mathbf{u}_{i}$. The corresponding (first-order accurate) FV discretization of (3) is then given by

$$
\mathbf{u}_{i}^{n+1}=\mathbf{u}_{i}^{n}-\frac{\Delta t}{\Delta x}\left[\mathbf{F}_{i+1 / 2}^{x}-\mathbf{F}_{i-1 / 2}^{x}\right]+\Delta t \mathbf{S}_{i} .
$$

We will restrict ourselves to these linear reconstruction methods in what follows.

\section{Flux formulae}

The generic algorithm (4) requires some prescription for the interface fluxes $\mathbf{F}_{i \pm 1 / 2}^{x}$. A straightforward calculation shows that the simple average

$$
F_{i+1 / 2}=\frac{1}{2}\left(F_{i}+F_{i+1}\right)
$$

makes (4) fully equivalent to the standard second order FD approach. As it is well known, this choice is prone to developing high-frequency noise in presence of steep gradients, like the ones appearing in black hole simulations. For this reason, artificial viscosity terms are usually required in order to suppress the spurious high-frequency modes [4].

We will consider here more general flux formulae, namely

$$
F_{i+1 / 2}=f\left(u_{L}, u_{R}\right),
$$

where $u_{L}, u_{R}$ stand for the left and right predictions for the dynamical field $u$ at the chosen interface (arrows and dots, respectively, in Fig. 1). In the (piecewise) linear case, they are given by

$$
u^{L}=u_{i}+1 / 2 \sigma_{i} \Delta x \quad u^{R}=u_{i+1}-1 / 2 \sigma_{i+1} \Delta x,
$$

where $\sigma_{i}$ stands for the slope of the chosen field in the corresponding cell.

A sophisticated choice is provided by the 'shockcapturing' methods (see Ref. [5] for a review). The idea is to consider the jump at the interface as a physical one (not just a numerical artifact). The characteristic decomposition of (the principal part of) the system is then used in order to compute some physically sound interface Flux. These advanced methods have been common practice in Computational Fluid Dynamics since decades. They were adapted to the Numerical Relativity context fifteen years ago [9], for dealing with the spherically symmetric (1D) black-hole case. They are still currently used in Relativistic Hydrodynamics codes, but their use in 3D black hole simulations has been limited by the computational cost of performing the characteristic decomposition of the evolution system at every single interface.

More recently, much simpler alternatives have been proposed, which require just the knowledge of the characteristic speeds, not the full characteristic decomposition. Some of them have yet been implemented in Relativistic Hydrodynamics codes [10]. Maybe the simplest choice is the local Lax-Friedrichs (LLF) flux formula [11]

$$
f\left(u_{L}, u_{R}\right)=\frac{1}{2}\left[F_{L}+F_{R}+c\left(u_{L}-u_{R}\right)\right],
$$

where the coefficient $c$ depends on the values of the characteristic speeds at the interface, namely:

$$
c=\max \left(\lambda_{L}, \lambda_{R}\right),
$$

where $\lambda$ is the spectral radius (the absolute value of the biggest characteristic speed).

When comparing the LLF choice (8) with the centered FD one (5), we can see that the supplementary terms play the role of a numerical dissipation. In this sense, a much more dissipative choice would be

$$
c=\frac{\Delta x}{\Delta t},
$$

which corresponds to (a piecewise linear generalization of) the original Lax-Friedrichs algorithm. Note that in any case the values of the dissipation coefficients are prescribed by the numerical algorithms: they are no arbitrary parameters, like in the FD case.

\section{FLUX SPLITTING APPROACH}

In the flux formulae approach (6), the information coming from both sides is processed at every interface, where different components are selected from either side in order to build up the flux there. We will consider here an alternative approach, in which the information is processed instead at the grid nodes, by selecting there the components of the flux that will propagate in either direction (flux splitting approach) [5]. 
The flux-splitting analogous of the original LLF formula (8, 9) can be obtained by splitting the flux into two simple components

$$
F_{i}^{ \pm}=F_{i} \pm \lambda_{i} u_{i}
$$

where $\lambda$ will be again the spectral radius at the given grid point. Each component is then reconstructed separately, leading to one-sided predictions at the neighbor interfaces. The final interface flux will be computed then simply as

$$
F_{i+1 / 2}=\frac{1}{2}\left(F_{L}^{+}+F_{R}^{-}\right)
$$

This method can also be expressed as a modified LLF flux formula, namely

$$
f\left(u_{L}, u_{R}\right)=\frac{1}{2}\left[F_{L}+F_{R}+\lambda_{L} u_{L}-\lambda_{R} u_{R}\right] .
$$

The main difference between the original LLF flux formula (8) and the flux-splitting variant (13) is that in the last case there is a clear-cut separation between the contributions coming from either the left or the right side of the interface, as it can clearly be seen in (12). In this way, one has a clear vision of the information flux in the numerical algorithm. The information from $F^{+}$components propagates in the forward direction, whereas the one from $F^{-}$components propagates backwards. This simple splitting provides in this way some insight that can be useful for setting up suitable boundary conditions. Moreover, it opens the door to using different slopes for the reconstruction of each flux component. We will see below how to take advantage of this fact in order to improve space accuracy.

\section{Third order accuracy}

As it is well known, the use of a consistent piecewiselinear reconstruction results generically into a secondorder space accuracy. A convenient choice is given by the centered slope

$$
\sigma^{C}=\frac{1}{2 \Delta x}\left(u_{i+1}-u_{i-1}\right)
$$

This is a good default choice (Fromm choice [5]), leading to reliable second-order accurate algorithms .

More general second-order algorithms can be obtained by replacing the centered slope $\sigma^{C}$ by any convex average of the left and right slopes,

$$
\sigma^{L}=\left(u_{i}-u_{i-1}\right) / \Delta x, \quad \sigma^{R}=\left(u_{i+1}-u_{i}\right) / \Delta x .
$$

In some applications, however, second order accuracy is not enough. The leading (third order) error is of the dispersion type, affecting the numerical propagation speeds. In the FD approach, this can be improved by using a fourth-order-accurate algorithm in combination with a fourth-order artificial dissipation term (which constitutes itself the leading error term). The resulting combination is third-order accurate.

In the standard FV approach, the standard way of getting (piecewise) third-order accuracy would be instead to replace the piecewise linear reconstruction by a piecewise parabolic one. The prototypical example is provided by the well known piecewise parabolic methods (PPM). The main complication of this strategy is that node values would no longer represent the cell averages of a given dynamical field. This would increase the complexity of the reconstruction process and the computational cost of the resulting algorithm.

There is a much simpler alternative, which takes advantage of the Flux splitting (11). The idea is to consider the resulting one-sided components $F^{ \pm}$as independent dynamical fields, each one with its own slope. The surprising result is that the choice

$$
\sigma^{+}=\frac{1}{3} \sigma^{L}+\frac{2}{3} \sigma^{R}, \quad \sigma^{-}=\frac{2}{3} \sigma^{L}+\frac{1}{3} \sigma^{R}
$$

leads, after the recombination (12), to a third-order accurate algorithm. The coefficients in (16) are unique: any other combination leads just to second-order accuracy.

Note that we are getting in this way third-order accuracy with a piecewise linear reconstruction (see the convergence test in Fig. 5 for a confirmation). This important result seems to be a peculiarity of the Fluxsplitting approach. In order to better understand it, let us suppress for a moment the lambda terms in (11,13). A straightforward calculation shows that, when using the slopes (16), the resulting algorithm coincides exactly with the standard fourth-order-accurate FD algorithm. Adding the lambda terms improves the stability of the algorithm at the price of downgrading the space accuracy to third order. This is precisely the same effect that the Kreiss-Oliger dissipation terms produce in the FD case. This confirms our result and suggests the interpretation of the algorithm (11, 13) as providing an adaptive generalization of the standard dissipation terms.

\section{THE 1D BLACK HOLE}

As a first test, let us consider the Schwarzschild Black Hole in spherical coordinates. We will write the line element in the 'wormhole' form:

$$
\mathrm{d} s^{2}=-(\tanh \eta)^{2} d t^{2}+4 m^{2}(\cosh \eta / 2)^{4}\left(d \eta^{2}+d \Omega^{2}\right),
$$

which can be obtained from the isotropic form by the following coordinate transformation

$$
r=m / 2 \exp (\eta) .
$$

The wormhole form (17) exploits the presence of a minimal surface (throat) at $\eta=0$. It is manifestly invariant by the reflection isometry

$$
\eta \leftrightarrow-\eta
$$




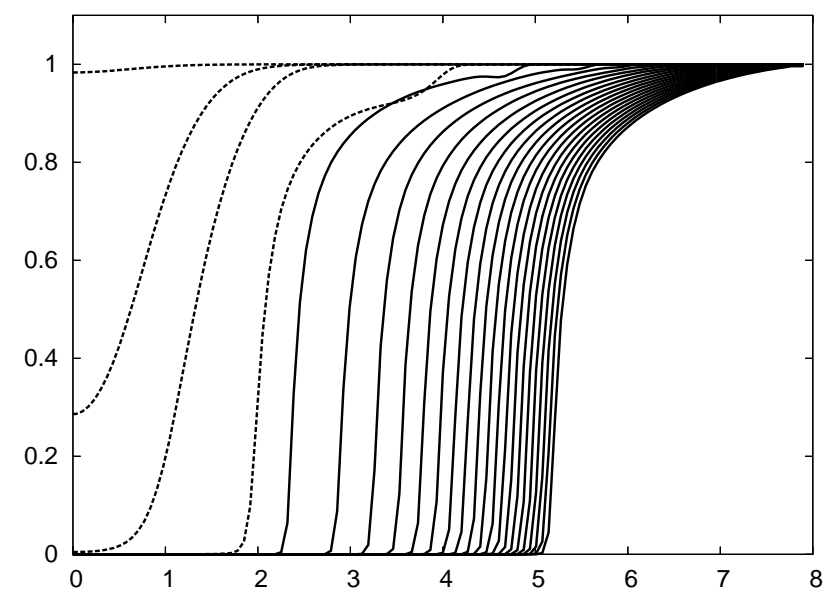

FIG. 2: Long-term FV simulation of a 1D black hole, with a single mesh of 120 gridpoints. The evolution of the lapse is shown up to $1000 \mathrm{~m}$, in intervals of $50 \mathrm{~m}$ (solid lines). The dotted lines correspond to $1 \mathrm{~m}, 3 \mathrm{~m}, 5 \mathrm{~m}$ and $25 \mathrm{~m}$. Note that the plots tend to cumulate at the end, due to the exponential character of the grid, as given by (18). No slope limiters have been used in this simulation.

so that the numerical simulations can be restricted to positive values of $\eta$. The isometry (19) provides a very convenient boundary condition at the throat. Moreover (18) implies

$$
d r=r d \eta
$$

so that an evenly spaced grid in $\eta$ corresponds to a geometrically increasing spacing in $r$. We can perform in this way long term simulations with a single grid of a limited size, as we will see below. This also allows to apply the standard boundary conditions in FV methods: two 'ghost' points are added by just copying the nearest neighbor values (or their time variation) for every dynamical field. The separation between incoming and outgoing information is automatically performed by the flux-splitting algorithm, so that boundary points are not special in this respect.

The simulations are performed with a spherically symmetric version of the Z3 formalism [14], as detailed in Appendix C. The free parameter $n$, governing the coupling with the energy constraint, is taken with unit value by default, but other similar values can be taken without affecting significatively the results, like $n=4 / 3$, which corresponds to the CADM case [15]. Regarding gauge conditions, we are using the generalized harmonic prescription for the lapse [16]

$$
\left(\partial_{t}-\mathcal{L}_{\beta}\right) \alpha=-f \alpha^{2} \operatorname{tr} K
$$

with zero shift (normal coordinates). We take a constant (unit) value of the lapse as initial data. We can see in Fig. 2 the evolution of the lapse in a long-term simulation (up to $1000 m$ ). We have chosen in this case $f=2 / \alpha$ (corresponding to the $1+\log$ slicing), but similar results

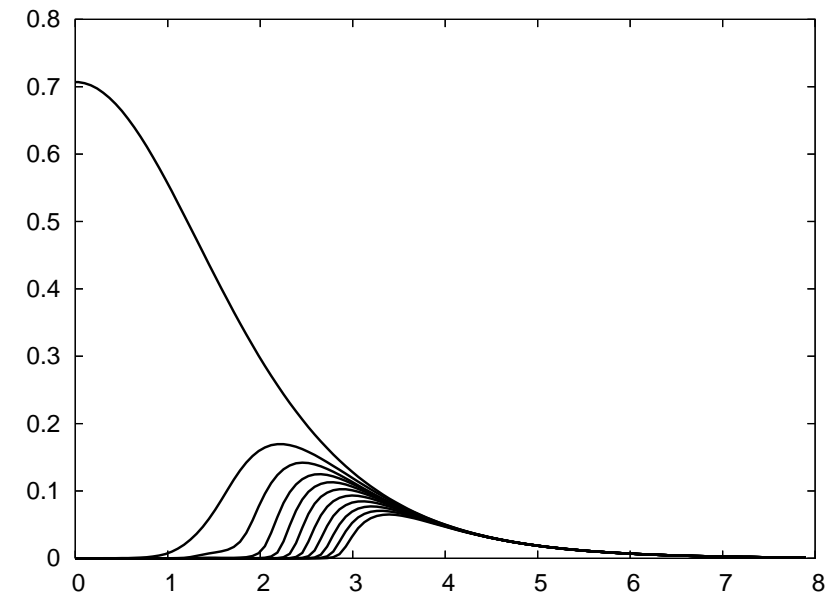

FIG. 3: The evolution of the propagation speed is shown up to $100 \mathrm{~m}$, in intervals of $10 \mathrm{~m}$, for the same simulation as in Fig. 2] The maximum values are clearly seen to decrease in time. Note the exponentially decreasing tail, as a result of the choice of the radial coordinate.

can be obtained with many other combinations of the form

$$
f=a+b / \alpha,
$$

where $a$ and $b$ are constant parameters.

Note that no slope limiters have been used in the simulation shown in Fig. 2. This can seem surprising at the first sight, but it can be better understood by looking at the propagation speed profiles shown in Fig. 3. The maximum propagation speed values decrease with time, due to the lapse collapse in the black hole interior region. This happens because the initial speed profile is exponentially decreasing with the chosen radial coordinate. The same decreasing arises for gauge speed. As a result, the Courant stability condition becomes less and less restrictive as the simulation proceeds, allowing us to take bigger timesteps. We have preferred instead to keep the initial timestep for the sake of accuracy. As far as all derivative terms get multiplied by $\Delta t$ in the algorithm (4), this gives us an extra safety factor that allows us to avoid using slope limiters.

As an accuracy check, we monitor the mass function [17], which is to be constant in space and time for the Schwarzschild case, independently of the coordinate system. In Fig. 团 we compare (the $L_{2}$ norm of) the errors in the mass function between a third-order FV simulation (without slope limiters) and the corresponding FD simulation (including a fourth order dissipation term like the one in ref. [3] with $\epsilon=0.015)$. We see that the FD method shows bigger errors at late times. One can argue that the leading error in the FD simulation is given by the dissipation terms, so that one can modify the result by lowering the numerical dissipation coefficient. However, lowering the viscosity coefficient used in Fig. 4, would result into a premature code crashing, like the one shown 


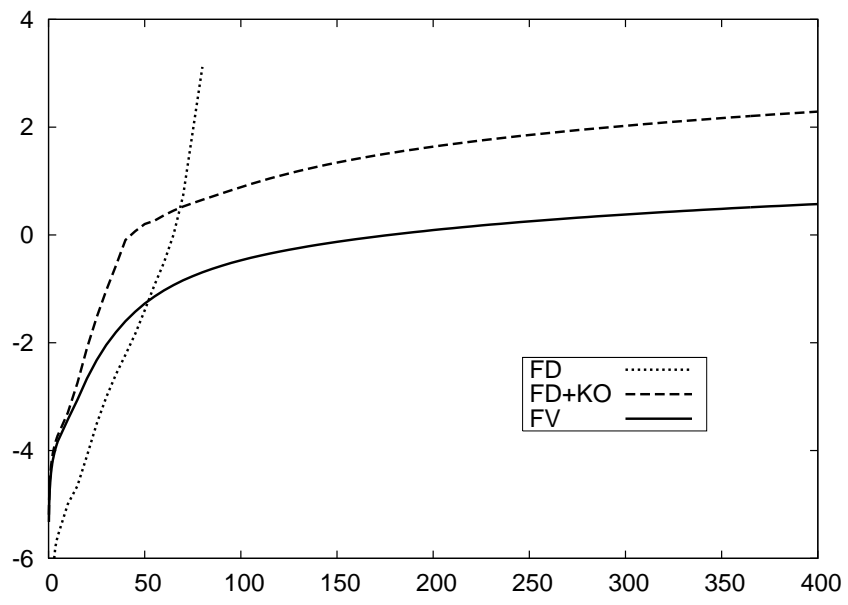

FIG. 4: Time evolution of the error in the mass function (logarithm of the $L_{2}$ norm) for three different numerical algorithms. The strictly fourth-order FD method, without extra dissipation terms, is the most accurate as expected, but crashes after a short time (measured in units of $m$ ). The other two algorithms (third-order accurate) get similar errors at early times, but the FV one performs much better in the long term than the FD with standard Kreiss-Oliger dissipation. The dissipation coefficient has been taken as low as allowed by code stability (see the text). All simulations were obtained with a single mesh of 120 gridpoints and using the $1+\log$ slicing prescription.

in the Figure for a strictly fourth order FD run, without the artificial dissipation term.

We can understand the need for dissipation by looking at the sharp collapse front in Fig. 2. We know that this is not a shock: it could be perfectly resolved by increasing the grid resolution as needed. In this way we can actually get long-term $1 \mathrm{D}$ black hole simulations, with a lifetime depending on the allowed resolution. This 'brute force' approach, however, can not be translated into the 3D case, where a more efficient management of the computational resources is required. This is where dissipation comes into play, either the numerical dissipation built in FV methods or the artificial one which is routinely added to fourth-order FD methods. Dissipation is very efficient in damping sharp features, corresponding to high-frequency Fourier modes. As a result, the collapse front gets smoothed out and can be resolved without allocating too many grid points. However, the more dissipation the more error. In this sense, Fig. 4 shows that adaptive viscosity built in the proposed FV method provides a good compromise between accuracy and computational efficiency.

Note that the error comparison is independent of the selected resolution. This is because the two stable methods in Fig. 4 are of third order accuracy, as confirmed by the local convergence test shown in Fig. 5 (solid line, corresponding to $t=10 \mathrm{~m}$ ). In the long term, however, large errors develop around the collapse front, downgrading the local convergence rate in the neighbor regions (dashed

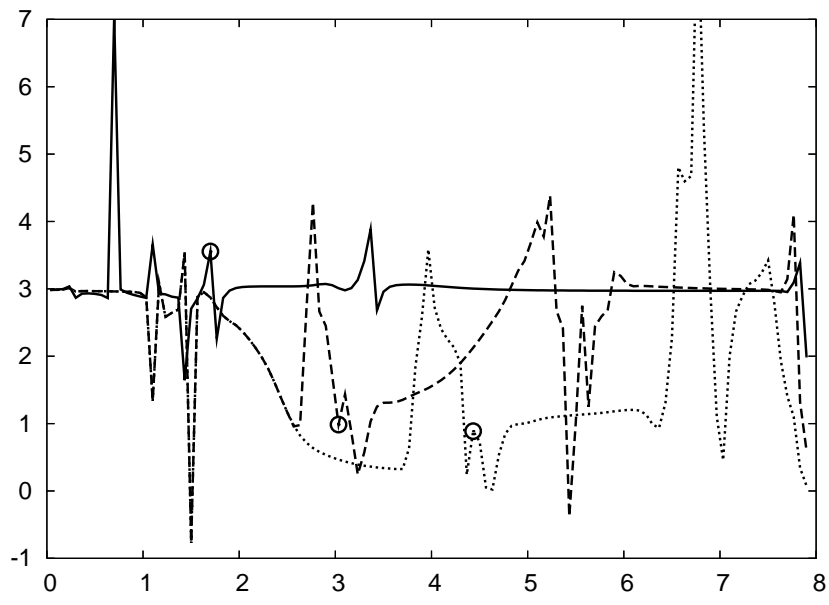

FIG. 5: Local convergence evolution for the mass function in a $1 \mathrm{D}$ black hole simulation. We can see the predicted thirdorder accuracy, when using the proposed slopes (16), around $t=10 m$ (solid line). At $t=100 m$ (dashed line), we yet see the downgrade in the regions around the collapse front (the apparent horizon position is marked with a circle). As the collapse front propagates (dotted line, corresponding to $t=400 \mathrm{~m}$ ), we can see the growth of the affected regions, specially the one behind the front.

and dotted lines in Fig. [5. corresponding to $t=100 \mathrm{~m}$ and $t=400 \mathrm{~m}$, respectively). This can not be seen as a failure of the algorithm properties, but rather as consequence of large errors in a highly non-linear context. This also shows that in simulations oriented to compute gravitational wave patterns (not the case of this paper), the waveform extraction zone must be safely located, away both from the outer boundary and from the collapse front.

\section{PRELIMINARY 3D RESULTS}

The 1D algorithm (4) can be easily adapted to the full three-dimensional (3D) case:

$$
\begin{aligned}
\mathbf{u}_{\{i j k\}}^{n+1}=\mathbf{u}_{\{i j k\}}^{n} & -\frac{\Delta t}{\Delta x}\left[\mathbf{F}_{\{i+1 / 2 j k\}}^{x}-\mathbf{F}_{\{i-1 / 2 j k\}}^{x}\right] \\
& -\frac{\Delta t}{\Delta y}\left[\mathbf{F}_{\{i j+1 / 2 k\}}^{y}-\mathbf{F}_{\{i j-1 / 2 k\}}^{y}\right] \\
& -\frac{\Delta t}{\Delta z}\left[\mathbf{F}_{\{i j k+1 / 2\}}^{z}-\mathbf{F}_{\{i j k-1 / 2\}}^{z}\right] \\
& +\Delta t \mathbf{S}_{\{i j k\}} .
\end{aligned}
$$

The structure of (23) suggests dealing with the 3D problem as a simple superposition of 1D problems along every single space direction. The stability analysis in Appendix A can then be extended in a straightforward way, showing that the strong stability requirement leads to a more restrictive upper bound on the timestep (in our case, using a cubic grid, this amounts to an extra $1 / 3$ factor). 


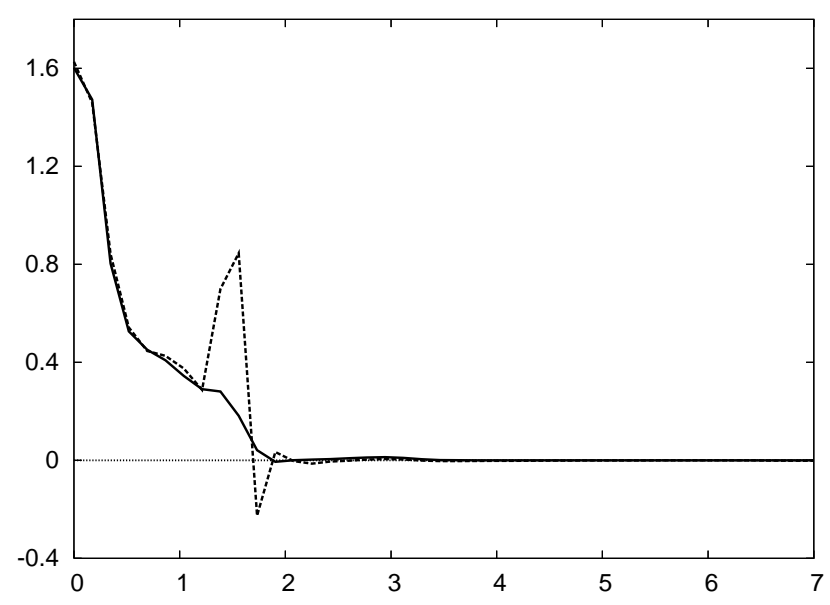

FIG. 6: Plot of the trace of the extrinsic curvature at $t=12 \mathrm{~m}$ for a low resolution simulation. The dotted line corresponds to the trace obtained by contraction from the individual components $K_{i j}$. The solid line is the same quantity computed directly as a primitive variable. The big difference corresponds to the transition between the collapsed and uncollapsed regions, where the lapse shows a steep profile

In cartesian-like coordinates, it is not so easy to take advantage of the reflection isometry (19). For this reason, we will evolve both the black-hole exterior and the interior domains. We can not use the $\eta$ coordinate for this purpose, because the symmetry center would correspond to $\eta \rightarrow \infty$. We will take instead the initial space metric in isotropic coordinates, namely

$$
\mathrm{d} l^{2}=\left(1+\frac{m}{2 r}\right)^{4} \delta_{i j} d x^{i} d x^{j} .
$$

We will replace then the vacuum black-hole interior by some singularity-free matter solution. To be more specific, we will allow the initial mass to have a radial dependence: $m=m(r)$ in the interior region. This allows to match a scalar field interior metric to (24) ('stuffed black-hole' approach [18]). The price to pay for using a regular metric inside the horizon is to evolve the matter content during the simulation: we have chosen the scalar field just for simplicity.

We have performed then a low-resolution simulation $(\Delta x=0.1 m)$ in order to monitor the errors in $\operatorname{tr} K$, which determines the evolution of the lapse. We see in Fig. 6] the comparison between the trace computed by contracting the individual $K_{i j}$ components (dotted line) and an auxiliary variable $K$ which is evolved by using the analytical equation for $\operatorname{tr} K$ (solid line). The difference is striking, even at the early time of the plot $(t=12 \mathrm{~m})$. Note the negative peak in the computed $\operatorname{tr} K$, which will produce a spike in the lapse leading to a premature code crashing.

This behavior could be somehow anticipated from our previous 1D simulations. The plots shown in Fig. 2 correspond to the mixed indices equations displayed in Appendix $\mathrm{C}$. We have performed for comparison the same simulations with 'downstairs' indices and the results look different. We actually double-checked both codes before realizing that just raising one index can make a difference at a given resolution. Of course, in $1 \mathrm{D}$ we can always increase resolution at will and verify that the two results get close enough. But this would be prohibitive in 3D, at least for single-grid simulations. Moreover, in $3 \mathrm{D}$ we have the additional difficulty of modelling curved features in a Cartesian grid. In the spherical case, the worst situation shows up along the main diagonal, precisely the view shown in Fig. 6.

These considerations can explain why the CADM formalism, which actually uses $\operatorname{tr} K$ as a primitive variable, has shown to be more robust even in single-grid simulations. This also explains why the use of a conformal decomposition was crucial in the 3D simulations performed with the old (non-covariant) Bona-Massó formalism in paper I, which used shock-capturing methods. The Z3 formalism can be interpreted as a covariant version of the same, but our results strongly suggest that the key element for robustness is not covariance but the use of a conformal decomposition.

As a final remark, let us focus on the boundary conditions implementation. The 3D FV algorithm (23) allows to apply the ghost point technique exactly in the same way as in the $1 \mathrm{D}$ case: by just copying (the time variation of) all the quantities from the neighbor interior point. There is no need for any special treatment for corners or vertices. Moreover, the simple FV methods presented here do not require the explicit use of the characteristic decomposition, not even at the boundaries. In spite of these simplifications, the robust stability test for the combined initial-boundary problem gives results equivalent to the ones obtained with maximally dissipative boundary conditions in a finite difference context (see Appendix B in Ref. [19] for details).

Acknowledgements: We acknowledge the hospitality of the Physics Department at the Louisiana State University during the final stage of this work. We are specially indebted with Dr. Palenzuela-Luque fur useful discussions. This work has been supported by the Spanish Ministry of Science and Education through the research project number FPA2004-03666 and by the Balearic Conselleria d'Economia Hissenda i Innovació through the project PRDIB-2005GC2-06.

\section{Appendix A: Stability and Monotonicity}

Let us assume that (the principal part of) the evolution system is strongly hyperbolic. This means that, for any chosen direction, we can express the system as a set of simple advection equations for the characteristic variables (eigenfields). In order to verify the stability properties of the proposed algorithms, it will be enough to consider a single advection equation with a generic speed $v$. The corresponding Flux will be given then by

$$
F(u)=v u .
$$


We will consider in the first place the first-order accurate approximation, obtained by a piecewise constant reconstruction (zero slope). The corresponding discretization can be obtained by replacing the prescription (12) into the general expression (44). The result is the linear three-point algorithm:

$$
\begin{aligned}
u_{i}^{n+1}=u_{i}^{n} & +\frac{\Delta t}{\Delta x}\left[\frac{1}{2}\left(\lambda_{i+1}-v_{i+1}\right) u_{i+1}^{n}\right. \\
& \left.+\frac{1}{2}\left(\lambda_{i-1}+v_{i-1}\right) u_{i-1}^{n}-\lambda_{i} u_{i}^{n}\right] .
\end{aligned}
$$

Allowing for the fact that $\lambda$ is chosen at every point as the absolute value of the maximum speed, we can see that all the $u^{n}$ coefficients are positive provided that the Courant stability condition

$$
\lambda \frac{\Delta t}{\Delta x} \leq 1
$$

is satisfied. Note however that a more restrictive condition is obtained in the three-dimensional case, where we must add up in (26) the contributions from every space direction.

As it is well known, the positivity of all the coefficients ensures that the algorithm is monotonicity-preserving, so that spurious numerical oscillations can not appear. This implies stability, but the converse is not true, as it is well known. Let us remember at this point that the centered FD discretization could be recovered from (26) simply by setting $\lambda$ to zero, although we would lose the monotonicity property in this way.

The monotonicity properties of the piecewise constant reconstruction are not ensured in the piecewise linear case. We can clearly see in Fig. 1 that monotonicity problems can arise in steep gradient regions. The reason is that either the series of left $\left\{u^{L}\right\}$ or right $\left\{u^{R}\right\}$ interface predictions can show spurious peaks which where not present in the original function. In the case of the centered slope (5), a detailed analysis shows that this will happen at a given interface only if the left and right slopes differ by a factor of three or more. This gives a more precise sense to the 'steep gradient' notion in the centered slopes case.

The natural way to remedy this is to enforce that both (left and right) interface predictions are in the interval limited by the corresponding left and right point values (interwinding requirement). This amounts to using the 'limited' slopes

$$
\sigma^{l i m}=\operatorname{minmod}\left(2 \sigma^{L}, \sigma, 2 \sigma^{R}\right),
$$

where $\sigma$ is the default slope at the given cell. This interwinding requirement is not enough, however, to ensure the positivity of all the coefficients in the resulting algorithm. A detailed analysis shows that an extra factor in the Courant condition would be required for monotonicity in this case:

$$
\lambda \frac{\Delta t}{\Delta x} \leq 1 / 2 .
$$

Note however that we are analyzing here the elementary step (44). This is just the building block of the time evolution algorithm. The exact stability and monotonicity limits for the time step would depend on the specific choice of the full time evolution algorithm [4], which will be described in Appendix B.

A word of caution must be given at this point. It is well known that the monotonicity results hold only for strictly Flux-conservative algorithms. This is not our case: the Source terms play an important physical role. Of course, these terms do not belong to the principal part, so that positivity of the Flux terms ensures some strong form of stability. Nevertheless, one must be very careful with the physical interpretation, because the first-order constraints (11) preclude any clear-cut isolation of the Source terms. This makes the analogy with Fluid Dynamics only approximative and the use of the slope limiters a risky matter: we could be removing in the Flux part some features that are required to compensate something in the Source part. Our experience is that, at least for smooth profiles, more robust numerical simulations are obtained when the slope limiters are switched off. The high frequency modes are kept under control by the numerical dissipation built in the proposed FV methods.

\section{Appendix B: Time accuracy}

The simple step (4) is only first-order accurate in time, and this fact is not changed by any of the space accuracy improvements we have considered up to now. The standard way of improving time accuracy is by the method of lines (MoL, see refs. [12] [4]). The idea is to consider (41) as a basic evolution step

$$
E\left(u^{n}, \Delta t\right)
$$

in order to build higher order algorithms. A convenient choice for these time evolution algorithms is provided the standard Runge-Kutta methods [13] (see also [4]). For instance, second order accuracy can be obtained in two steps:

$$
u^{*}=E\left(u^{n}, \Delta t\right) \quad u^{n+1}=\frac{1}{2}\left[u^{n}+E\left(u^{*}, \Delta t\right)\right],
$$

and third-order time accuracy with one more intermediate step:

$$
\begin{aligned}
u^{* *} & =\frac{3}{4} u^{n}+\frac{1}{4} E\left(u^{*}, \Delta t\right) \\
u^{n+1} & =\frac{1}{3} u^{n}+\frac{2}{3} E\left(u^{* *}, \Delta t\right) .
\end{aligned}
$$

Note that the positivity of all the coefficients in (31, 32) ensures that the monotonicity property of the basic step (30) will be preserved by the resulting strong-stabilitypreserving (SSP) algorithm. This interesting property comes at the price of keeping the upper limit on $\Delta t$ that is required for the monotonicity of the basic step. This 
is a clear disadvantage with respect to the case in which the standard FD approach is being used for space discretization, in which one is only limited by plain stability, not monotonicity. Then, there are Runge-Kutta algorithms (with non-positive coefficients) that alow to take $\Delta t$ larger than the one required by the standard Courant condition [4].

Conversely, second order Runge-Kutta algorithms like (31) are unstable when used in combination with FD space discretization, unless artificial dissipation is added in order to recover stability (not just monotonicity) [4]. This is why FD simulations currently use at least a thirdorder time evolution algorithm.

\section{Appendix C: Z3 evolution equations}

The Z3 evolution system [14, 15] is given by:

$$
\begin{aligned}
\left(\partial_{t}\right. & \left.-\mathcal{L}_{\beta}\right) \gamma_{i j}=-2 \alpha K_{i j} \\
\left(\partial_{t}\right. & \left.-\mathcal{L}_{\beta}\right) K_{i j}=-\nabla_{i} \alpha_{j}+\alpha\left[R_{i j}+\nabla_{i} Z_{j}+\nabla_{j} Z_{i}\right. \\
& \left.-2 K_{i j}^{2}+\operatorname{tr} K K_{i j}-S_{i j}+\frac{1}{2}(\operatorname{tr} S+(n-1) \tau) \gamma_{i j}\right] \\
& -\frac{n}{4} \alpha\left[\operatorname{tr} R+2 \nabla_{k} Z^{k}\right. \\
& \left.\quad+4 \operatorname{tr}^{2} K-\operatorname{tr}\left(K^{2}\right)-2 Z^{k} \alpha_{k} / \alpha\right] \gamma_{i j} \\
\left(\partial_{t}-\right. & \left.\mathcal{L}_{\beta}\right) Z_{i}=\alpha\left[\nabla_{j}\left(K_{i}{ }^{j}-\delta_{i}{ }^{j} \operatorname{tr} K\right)-2 K_{i}{ }^{j} Z_{j}-S_{i}\right],
\end{aligned}
$$

where $n$ is an arbitrary parameter governing the coupling of the energy constraint.

The fully first-order version can be obtained in the standard way, by introducing the additional fields

$$
D_{k i j} \equiv \frac{1}{2} \partial_{k} \gamma_{i j}
$$

Note that the ordering constraint (11) reads

$$
\partial_{r} D_{k i j}=\partial_{k} D_{r i j},
$$

which is no longer an identity for the first order system. As a consequence of this ordering ambiguity of second derivatives, the Ricci tensor term in (the first order version of) the evolution equation (34) can be written in many different ways. Then, an ordering parameter $\zeta$ can be introduced [15], so that the parameter choice $\zeta=+1$ corresponds to the standard Ricci decomposition

$$
{ }^{(3)} R_{i j}=\partial_{k} \Gamma^{k}{ }_{i j}-\partial_{i} \Gamma_{k j}^{k}+\Gamma_{r k}^{r} \Gamma^{k}{ }_{i j}-\Gamma_{r i}^{k} \Gamma_{k j}^{r}
$$

whereas the opposite choice $\zeta=-1$ corresponds instead to the decomposition

$$
\begin{aligned}
{ }^{(3)} R_{i j} & =-\partial_{k} D^{k}{ }_{i j}+\partial_{(i} \Gamma_{j) k}{ }^{k}-2 D_{r}{ }^{r k} D_{k i j} \\
& +4 D^{r s}{ }_{i} D_{r s j}-\Gamma_{i r s} \Gamma_{j}{ }^{r s}-\Gamma_{r i j} \Gamma_{k}^{r k}
\end{aligned}
$$

which is most commonly used in Numerical Relativity codes. We can then consider the generic case as a linear combination of (38) and (39).

In the spherically symmetric vacuum case, the first order version of the system (33) 34 is free of any ordering ambiguity. It can be written as

$$
\begin{aligned}
\partial_{t} \gamma_{r r}= & -2 \alpha \gamma_{r r} K_{r}{ }^{r}, \quad \partial_{t} \gamma_{\theta \theta}=-2 \alpha \gamma_{\theta \theta} K_{\theta}{ }^{\theta} \\
\partial_{t} K_{r}{ }^{r}+ & \partial_{r}\left[\alpha \gamma^{r r}\left(A_{r}+(2-n) D_{\theta}{ }^{\theta}-(2-n / 2) Z_{r}\right)\right]= \\
& \alpha\left[\left(K_{r}{ }^{2}\right)^{2}+(2-n) K_{r}{ }^{r} K_{\theta}{ }^{\theta}-(n / 2)\left(K_{\theta}{ }^{2}\right)^{2}\right. \\
& -\gamma^{r r} D_{r}{ }^{r}\left(A_{r}+(2-n) D_{\theta}{ }^{\theta}+(n / 2-2) Z_{r}\right) \\
& +\gamma^{r r} D_{\theta}{ }^{\theta}\left((2-n) A_{r}-(2-3 n / 2) D_{\theta}{ }^{\theta}-n Z_{r}\right) \\
& \left.-\gamma^{r r}(2-n) A_{r} Z_{r}-(n / 2) \gamma^{\theta \theta}\right] \\
\partial_{t} K_{\theta}{ }^{\theta}+ & \partial_{r}\left[\alpha \gamma^{r r}\left((1-n) D_{\theta}{ }^{\theta}+(n / 2) Z_{r}\right)\right]= \\
& \alpha\left[(1-n) K_{r}{ }^{r} K_{\theta}{ }^{\theta}+(2-n / 2)\left(K_{\theta}{ }^{\theta}\right)^{2}\right. \\
& -\gamma^{r r} D_{r}{ }^{r}\left((1-n) D_{\theta}{ }^{\theta}+(n / 2) Z_{r}\right) \\
& +\gamma^{r r} D_{\theta}{ }^{\theta}\left((2-n) Z_{r}-(2-3 n / 2) D_{\theta}{ }^{\theta}\right) \\
& \left.-n \gamma^{r r} A_{r}\left(D_{\theta}{ }^{\theta}-Z_{r}\right)+(1-n / 2) \gamma^{\theta \theta}\right] \\
\partial_{t} Z_{r}+ & \partial_{r}\left[2 \alpha K_{\theta}{ }^{\theta}\right]= \\
& 2 \alpha\left[D_{\theta}{ }^{\theta}\left(K_{r}{ }^{r}-K_{\theta}{ }^{\theta}\right)+A_{r} K_{\theta}{ }^{\theta}-K_{r}{ }^{r} Z_{r}\right] \\
\partial_{t} D_{r}{ }^{r}+ & \partial_{r}\left[\alpha K_{r}{ }^{r}\right]=0, \quad \partial_{t} D_{\theta}{ }^{\theta}+\partial_{r}\left[\alpha K_{\theta}{ }^{\theta}\right]=0,(43)
\end{aligned}
$$

where we are using normal coordinates (zero shift). The slicing condition (21) can be written as

$$
\partial_{t} \alpha=-\alpha^{2} f \operatorname{tr} K, \quad \partial_{t} A_{r}+\partial_{r}[\alpha f \operatorname{tr} K]=0 .
$$

The mass function can be defined for spherically symmetric spacetimes as [17]

$$
2 M=Y\left[1-g^{a b} \partial_{a} Y \partial_{b} Y\right]
$$

where $Y$ stands for the area radius. In spherical coordinates we get

$$
2 M(t, r)=\sqrt{\gamma_{\theta \theta}}\left\{1+\gamma_{\theta \theta}\left[\left(K_{\theta}{ }^{\theta}\right)^{2}-\gamma^{r r}\left(D_{\theta}{ }^{\theta}\right)^{2}\right]\right\} .
$$

The mass function has a clear physical interpretation: it provides the mass inside a sphere of radius $r$ at the given time $t$. It follows that $M(t, r)$ must be constant for the Schwarzschild spacetime, no matter which coordinates are being used. This provides a convenient accuracy check for numerical simulations. 
[1] M. Alcubierre and B. Brügmann, Phys. Rev. D63 104006 (2001).

[2] L. Baiotti, I. Hawke, L. Rezzolla and E. Schnetter, Phys. Rev. Lett. 94131101 (2005).

[3] L. Baiotti and L. Rezzolla, Phys. Rev. Lett. 97141101 (2006).

[4] B. Gustafson, H.O. Kreiss and J. Oliger, Time dependent problems and difference methods, Wiley, New York (1995).

[5] R. J. LeVeque, Finite Volume Methods for Hyperbolic Problems. Cambridge (Cambridge 2002).

[6] A. Arbona, C. Bona, J. Massó and J. Stela, Phys. Rev. D60 104014 (1999).

[7] T. Nakamura et al, Prog. Theor. Phys. Suppl. 90, 1 (1987).

[8] C. Bona and J. Massó, Phys. Rev. D40 1022 (1989).

[9] C. Bona and J. Massó, Phys. Rev. Lett. D68 1097 (1992).

[10] M. A. Aloy, J. A. Pons and J. M. Ibáñez, Computer
Physics Commun. 120115 (1999).

[11] V. V. Rusanov, J. Comput. Mat. Phys. USSR 1267 (1961).

[12] O. A. Liskovets, Differential equations I 1308-1323 (1965).

[13] S. Gottlieb, C.-W. Shu and E. Tadmor, SIAM Review 43, 89 (2001).

[14] C. Bona, T. Ledvinka and C. Palenzuela, Phys. Rev. D66 084013 (2002).

[15] C. Bona, T. Ledvinka, C. Palenzuela, M. Žáček, Phys. Rev. D69 064036 (2004).

[16] C. Bona, J. Massó, E. Seidel, and J. Stela, Phys. Rev. Lett. 75600 (1995).

[17] G. Lemaître, Rev. Mod. Phys. 21357 (1949).

[18] A. Arbona et al., Phys. Rev. D57 2397 (1998).

[19] C. Bona, T. Ledvinka, C. Palenzuela, M. Žáček, Class. Quantum Grav. 22 1-19 (2005). 